mechanism whereby SRF is activated by growth factors is unknown, but there are three ways in which it might work in principle. First, phosphorylation by growthfactor-activated kinases might induce DNA binding by promoting dimerization where the monomeric protein has only weak affinity for its binding site. This is very unlikely to apply to SRF which seems to dimerize without stimulation. Second, it might increase the ability of the protein to activate transcription without affecting its affinity for DNA. Third, it might promote the formation of a complex between the SRF dimer and a separate protein containing or comprising a transcriptional activating domain. This might seem a somewhat gratuitous suggestion but for some recent evidence, to which I shall return later, for the formation of functionally important complexes between at least some of the immediate early gene products themselves. The isolation by Triesman of the gene encoding SRF should accelerate the exploration of this and other possible modes of activation of the SRF protein.

Evidence for a contribution of at least two of these mechanisms to c-fos activation may be more rapidly forthcoming from investigations on its response to cyclic AMP, which induces c-fos transcrip-

\section{From egg to ascus}

Maturation-promoting factor (MPF) was first discovered as a cytoplasmic extract of mature Xenopus eggs that would, on injection into immature oocytes, induce entry into meiosis. It has since been demonstrated in the cytoplasm of both yeast and mammalian cells where it induces mitosis in the normal cell cycle. Although it was discovered in 1971, and has been known for some years to be a crucial component of cell-cycle regulation in mammalian cells, it has proved extraordinarily resistant to purification and its nature and mechanism of action have eluded precise definition.

There is thus an element almost of bathos in the evidence presented by David Beach (Cold Spring Harbor Laboratories) that MPF, or at least an essential component thereof, is a $34 \mathrm{~K}$ protein kinase identified last year ${ }^{1.2}$ as the product of the mammalian homologue of the cell-cycle gene $c d c 2$ of fission yeast. The story is as follows.

With Giulio Draetta, Beach has shown that the $34 \mathrm{~K}$ protein kinase (p34) of HeLa cells exists in an inactive complex with a second protein, $p 13$, until late in the G2 phase of the cell cycle, when its phosphorylation is increased and it becomes associated with a third protein (p62); at this stage it is activated, its kinase activity rising to a peak at the point of entry into mitosis. It thus shares with MPF the property of increased kinase activity without any increase in abundance.

The evidence for identity with MPF tion through a pathway that is independent of the serum-responsive element and is presumed to depend upon one or more of the sequences corresponding to defined cyclic-AMP-responsive elements (CREs) in the regulatory region of the gene (Ron Prywes, Rockefeller University; Inder Verma, Salk Institute; Michael Gilman, Cold Spring Harbor Laboratory).

Direct evidence for this pathway has been adduced from experiments by Karl Riabowol with Michael Gilman in James Feramisco's laboratory (Cold Spring Harbor Laboratory). Microinjection into cells of the catalytic subunit of the cyclicAMP-dependent protein kinase A leads to the activation of c-fos transcription, and the effect can be blocked by the co-injection of oligonucleotides corresponding to a cyclic-AMP-responsive element but not by a serum-responsive-element oligonucleotide. Investigations by Yamamoto et al. ${ }^{19}$ suggest how protein kinase $\mathrm{A}$ activated by cyclic AMP might activate proteins binding to cyclic-AMP-responsive elements (CREB) and reveal a complex picture of their regulation.

Using a CREB from brain, Verma and Yamamoto et al. have shown in gel-retardation assays that whereas the $43 \mathrm{~K}$ brain protein will bind to its cognate element in either the c-fos or the somatostatin gene, a

comes from investigations, done in collaboration with William Dunphy and John Newport, into the effect of the yeast p13 protein on the action of MPF on mitotic initiation in cell-free extracts of Xenopus eggs. It has been impossible to demonstrate directly that the product of the $c d c 2$ gene can promote entry of Xenopus nuclei into mitosis, but Beach reasons that this may be attributable to some failure to reproduce the appropriate phosphorylation and subunit composition with the heterologous protein. What he has now shown is that p13 added to cell-free extracts will block the effect of Xenopus MPF, partially purified by Lohka et al. ${ }^{3}$, and that MPF activity is retained by a p13 column, which moreover retains the Xenopus $c d c 2$ product, along with a $42 \mathrm{~K}$ protein that may be a second component of Xenopus MPF.

How far yeast cell-cycle genetics will now be able to clarify the control of proliferation in mammalian cells remains to be seen. Although the mammalian $c d c 2$ product will complement temperature-sensitive yeast mutants, this conservation of biochemical function does not necessarily reflect a precise conservation of physiological function: $c d c 2$ in fission yeast seems to be required in $\mathrm{G} 1$ and $\mathrm{G} 2$ rather than at mitosis; and the budding-yeast homologue, $c d c 28$, controls entry into G1.

I. Draetta, G., Brizuela, L., Potashkin, J. \& Beach. D. Cell 50, $319-325$ (1987).

Lee, M. \& Nurse, P. Vature 327, 31-35 (1987)

3. Lohka. M.L.. Hayes. M.K. \& Maller. J.L. Proc, natn. Acad. Sci. U.S.A. $85,3009-3013(1988)$. higher molecular weight complex (presumed to be a CREB dimer) binds with higher affinity. Experiments with protein kinases in vitro have shown that phosphorylation of the purified protein at two sites promotes dimerization, whereas phosphorylation at a third, separate site increases the ability of the protein to activate transcription. Only the increase in transcriptional activation is, however, attributable to phosphorylation by protein kinase A: unexpectedly, it is phosphorylation by protein kinase $\mathrm{C}$ - not normally associated with cyclic AMP-mediated signalling - - that promotes dimerization. While most of these experiments were done with the somatostatin CRE, it seems likely that similar mechanisms apply to the equivalent element in the c-fos gene.

The regulatory region of c-fos is complex and contains, as well as binding sites for serum-responsive and cyclic AMPdependent factors, at least one sequence corresponding to the specific binding site for AP-1, a factor now implicated in the effector functions of FOS. One of these is the regulation of its own production; and at Cold Spring Harbor two groups reported evidence implicating AP-1 in the mechanism of this auto-regulation.

\section{Suppressing the activators}

It is a feature common to the immediate early genes that their activation is not only rapid but transient; and the enhanced transcriptional activation of genes such as c-myc and c-fos under the inhibition of protein synthesis led very early on to the suggestion that they negatively regulate their own expression as well as (presumably) positively regulating the expression of other cellular genes. (It is this negative feedback that is believed often to be abrogated or overridden in oncogenic mutants at least of c-myc.)

Neither MYC nor FOS however has ever been shown to bind specifically to DNA, and their mechanism of action remained moot until recently when it transpired that the $39 \mathrm{~K}$ protein with which FOS is commonly associated is the mammalian transcription factor AP-1, which is the product of $c-j u n^{12.13 .20}$. The revelations that have followed this discovery apply in practice only to the function of FOS, but there are indirect suggestions of a similar role for MYC and other products of nuclear cellular oncogenes such as c- $m y b$.

Like c-fos, c-jun is rapidly induced by growth factors; in the absence of FOS, JUN binds weakly but specifically to the DNA binding site defined by its ability to bind AP-1; in the absence of JUN, FOS does not bind the AP-1 site at all. When both are present. FOS is found to associate with DNA containing the AP-1 binding site, and the specific binding affinity of JUN is increased (Tom Curran, Roche Institute, New Jersey; Robert Tjian, Berkeley). This suggests that FOS and 